\title{
A near-field error sensing strategy for compact multi-channel active sound radiation control in free field
}

Jiancheng Tao, Qin Guo, and Xiaojun Qiu

Citation: The Journal of the Acoustical Society of America 146, 2179 (2019); doi: 10.1121/1.5127179

View online: https://doi.org/10.1121/1.5127179

View Table of Contents: https://asa.scitation.org/toc/jas/146/4

Published by the Acoustical Society of America

\section{ARTICLES YOU MAY BE INTERESTED IN}

Deep transfer learning for source ranging: Deep-sea experiment results

The Journal of the Acoustical Society of America 146, EL317 (2019); https://doi.org/10.1121/1.5126923

Analysis of spherical isotropic noise fields with an A-Format tetrahedral microphone

The Journal of the Acoustical Society of America 146, EL329 (2019); https://doi.org/10.1121/1.5127736

A versatile computational approach for the numerical modelling of parametric acoustic array

The Journal of the Acoustical Society of America 146, 2163 (2019); https://doi.org/10.1121/1.5126863

Passive monitoring of nonlinear relaxation of cracked polymer concrete samples using acoustic emission

The Journal of the Acoustical Society of America 146, EL323 (2019); https://doi.org/10.1121/1.5127519

Acoustic surface wave generation over rigid cylinder arrays on a rigid plane

The Journal of the Acoustical Society of America 146, 2137 (2019); https://doi.org/10.1121/1.5126856

Classification of red hind grouper call types using random ensemble of stacked autoencoders

The Journal of the Acoustical Society of America 146, 2155 (2019); https://doi.org/10.1121/1.5126861

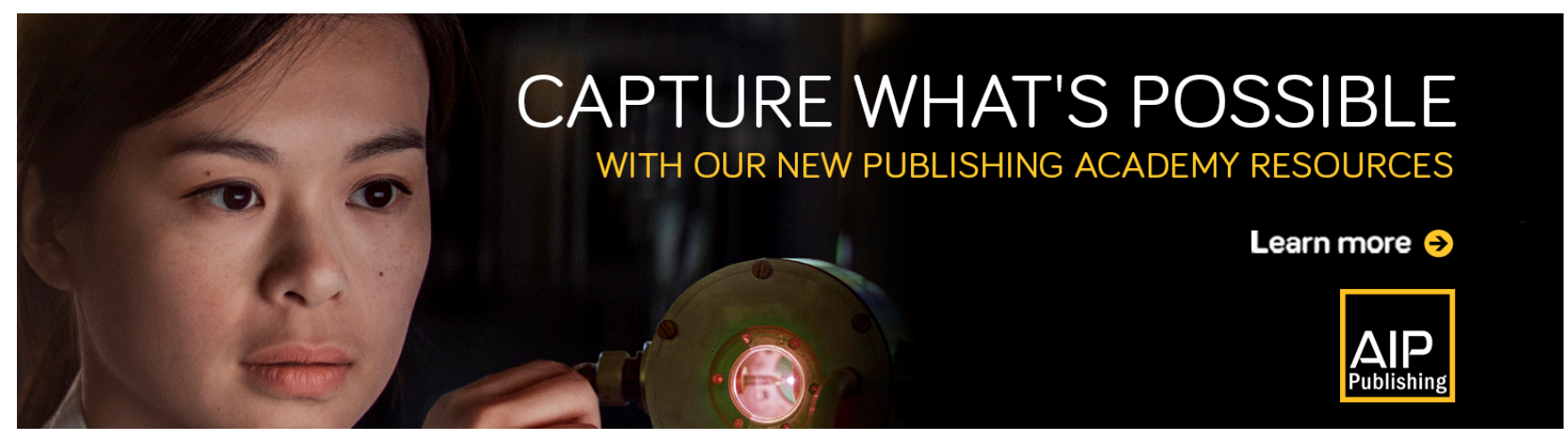




\title{
A near-field error sensing strategy for compact multi-channel active sound radiation control in free field
}

\author{
Jiancheng Tao, ${ }^{1, a)}$ Qin Guo, ${ }^{1}$ and Xiaojun Qiu ${ }^{2}$ \\ ${ }^{1}$ Key Laboratory of Modern Acoustics and Institute of Acoustics, Nanjing University, Nanjing 210093, China \\ ${ }^{2}$ Centre for Audio, Acoustics and Vibration, Faculty of Engineering and Information Technology, University of \\ Technology Sydney, New South Wales 2007, Australia
}

(Received 11 April 2019; revised 26 July 2019; accepted 9 September 2019; published online 3 October 2019)

\begin{abstract}
Noise reduction performance of a compact active sound radiation control system is significantly affected by locations of the error microphones which are required to be installed near the primary source. In this paper, near-field error sensing for multi-channel active radiation control systems in free field is investigated, and it is found that the optimal locations of error sensors for minimizing the sum of squared sound pressure are between the primary source and the secondary sources distributed uniformly on a sphere surface surrounding the primary source. Both simulation and experiment results show that the optimal locations of error microphones are independent of the type of primary source when there are sufficient secondary sources. These optimal locations remain unchanged at low frequencies and move toward secondary sources when the secondary source number increases. Therefore, for active radiation control applications in low frequency range, a compact multi-channel system can be developed by locating error microphones between the primary source and secondary sources. @ 2019 Acoustical Society of America. https://doi.org/10.1121/1.5127179
\end{abstract}

[JFL]

Pages: 2179-2187

\section{INTRODUCTION}

Active sound radiation control systems, which employ secondary sound sources and error sensors for global sound radiation reduction, have been extensively investigated for different noise sources such as transformers, fans, and gearboxes. ${ }^{1-4}$ The secondary sound sources are often placed as close as possible to the primary source to obtain high global noise reduction, so the compactness of such systems is mainly determined by the error sensing strategies. ${ }^{5}$

Several near-field error sensing strategies have been proposed for active sound radiation control. The near-field pressure and intensity distributions of the actively controlled plate have been studied analytically and the strategies for near-field sensors to minimize the far-field radiation power have been investigated. ${ }^{6}$ Four acoustic near-field sensing strategies for the active control of the sound field radiated from a monopole source were compared, and it was found that using the radial active intensity at a number of positions close to the primary source usually could give better sound power attenuation than using the squared pressure, acoustic kinetic energy density, or total acoustic energy density. ${ }^{7}$

The near-field sound intensity minimization does not always provide significant improvement as compared to the near-field squared pressure minimization in the active control of sound radiation from a vibrating plate due to the fact that the sum of the intensities can be driven to negative values with control when the intensity sensors are placed in the near-field of the primary source. ${ }^{8}$ The noise reduction performance was reported for the systems employing force actuators and acoustic error sensors to control the radiation from a

a)Electronic mail: jctao@nju.edu.cn plate and a small transformer, and it was found that better noise reduction could be achieved by minimizing the sum of sound intensity if a large number of acoustic error sensors are placed sufficiently close to the primary source., ${ }^{9,10}$

A near-field error sensing strategy was developed recently by minimizing the weighted sum of the sound pressure at a planar error microphone array near the primary source to reduce the sound radiation to a certain direction. ${ }^{11}$ It was found that the microphone spacing should be no more than half the wavelength of the control frequency and the minimum size of the error microphone array should be approximately twice the size of the primary source when the secondary source is close to the primary source.

The research mentioned above is on error sensing strategies which use either sound pressure, intensity or some kinds of transformation to estimate the sound radiation power in the whole space or in a certain direction. In every strategy, the error sensor location is a critical factor for the system performance. It is well known that the sound power reduction of a single channel system varies significantly with the error sensor location when the cost function is the near-field sound pressure and the optimal error sensor location is found to be at the point of the greatest acoustic pressure attenuation. ${ }^{5}$ For active control systems with two secondary sources and one error microphone, it was shown that the error microphone should be placed between the monopole primary source and one secondary source and the optimal distance ratio should be 0.618 for the best performance. ${ }^{12}$

The experimental results with a 4-channel active sound radiation control system demonstrated that placing the error sensors in the optimal near-field locations results in significant noise reduction and other error sensor displacements may also provide appreciable far-field attenuation considering the practical 
behavior of sound sources and error sensors. ${ }^{13}$ Genetic searching algorithms have been employed to optimize the locations of ten error microphones in an 8-channel active transformer noise control system to achieve global noise reduction at 100 and $200 \mathrm{~Hz}^{14}$ It is observed that the optimized locations of error microphones differ with the frequency.

The virtual error sensing techniques, which estimate the error signal at virtual target locations with local physical sensors, have been investigated in different applications of active noise control. The error microphones have been placed near the secondary sources to achieve noise reduction around the observer's ears in an active headset design, ${ }^{15}$ and near the top of a passive barrier to increase far filed noise reduction in an active noise barrier system. ${ }^{16}$ A moving zone of quiet has been effectively created in a one-dimensional duct for narrow band noise by exerting an adaptive virtual microphone technique, ${ }^{17}$ and the performance of the virtual microphone techniques was tested by experiments in the noise-reducing casings. ${ }^{18,19}$ To implement the virtual microphone techniques, prior system identification is required and the acoustic environment usually needs to be time invariant.

Despite all the research mentioned in the preceding text, little research report has been found on the near-field error sensing strategy for multi-channel active sound radiation control in free field. In this paper, the secondary sources are proposed to be evenly distributed around the primary source while the error microphones are proposed to be placed between the secondary sources and the primary source. The optimal locations of error microphones in this kind of systems for a monopole primary source are analyzed first, and then the dependence of the optimal locations on the frequency, the number of secondary sources and the type of primary sources is illustrated with numerical simulations. Finally, experimental results are presented to support the proposed near-field error sensing strategy.

\section{THEORY}

Figure 1 shows a multi-channel active radiation control system in free space, where $M$ secondary sources and $M$ error microphones are evenly distributed on two sphere surfaces respectively centered at a primary source with a strength of $Q_{\mathrm{p}}$. Each error microphone is on the line determined by its collaborated secondary source and the primary source, and the error microphones are placed between the primary source and secondary sources if the radius of the error microphone surface $d_{\mathrm{pe}}$ is smaller than the radius of the secondary source surface $d_{\mathrm{ps}}$.

The total sound pressure at the error microphones with control can be formulated as ${ }^{5,20}$

$$
\mathbf{P}_{\mathrm{e}}=\mathbf{Z}_{\mathrm{se}} \mathbf{Q}_{\mathrm{s}}+\mathbf{Z}_{\mathrm{pe}} Q_{\mathrm{p}}
$$

where $\mathbf{Z}_{\mathrm{se}}$ is an $M \times M$ matrix with the element on the $i$ th row and $j$ th column being $\mathrm{j} k \rho_{0} c_{0} e^{-\mathrm{j} k d_{\mathrm{se}, j i}} / 4 \pi d_{\mathrm{se}, j i}$ for point monopole secondary sources, $d_{\mathrm{se}, j i}$ is the distance between the $j$ th secondary source and $i$ th error microphone, $\rho_{0}$ is the air density, $c_{0}$ is the sound speed, $k=\omega / c_{0}$ is the wave number, $\omega$ is the angular frequency, $\mathbf{Q}_{\mathrm{s}}$ is the strength vector of

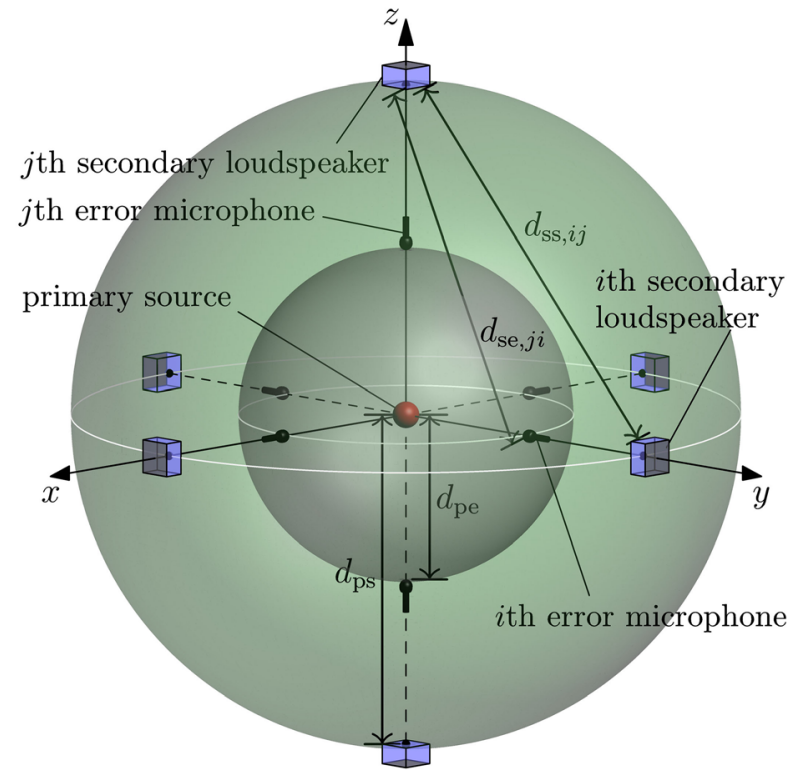

FIG. 1. (Color online) Sketch map of the active sound radiation control system with $M$ secondary sources and $M$ error microphones.

secondary sources, and $\mathbf{Z}_{\mathrm{pe}}$ is an $M \times 1$ vector with all elements being $\mathrm{j} k \rho_{0} c_{0} e^{-\mathrm{j} k d_{\mathrm{pe}}} / 4 \pi d_{\mathrm{pe}}$ for a point monopole primary source.

If the sum of squared sound pressure at error microphones is the cost function, the optimal strength vector of secondary sources can be obtained by equalizing the sound pressure at all error microphones in Eq. (1) to zero, so

$$
\mathbf{Q}_{\mathrm{s}}^{\mathrm{p}}=-\mathbf{Z}_{\mathrm{se}}^{-1} \mathbf{Z}_{\mathrm{pe}} Q_{\mathrm{p}}
$$

Because the secondary sources and error microphones are evenly distributed, the optimal strengths of all secondary sources are the same. Therefore, the optimal strength of the $i$ th secondary source can be expressed as

$$
Q_{\mathrm{s}, i}^{\mathrm{p}}==-Q_{\mathrm{p}} \frac{\mathrm{e}^{-\mathrm{j} k d_{\mathrm{pe}}}}{d_{\mathrm{pe}}}\left(\sum_{j=1}^{M} \frac{e^{-\mathrm{j} k d_{\mathrm{se}, j i}}}{d_{\mathrm{se}, j i}}\right)^{-1} .
$$

The total sound power with control can be formulated as ${ }^{5,20}$

$$
W=\mathbf{Q}_{\mathrm{s}}^{\mathrm{H}} \mathbf{A} \mathbf{Q}+\mathbf{Q}_{\mathrm{s}}^{\mathrm{H}} \mathbf{b}+\mathbf{b}^{\mathrm{H}} \mathbf{Q}_{\mathrm{s}}+W_{0},
$$

where $\mathbf{A}$ is an $M \times M$ matrix composed by the radiation resistance between two secondary sources, $b$ is an $M \times 1$ vector consisting of the mutual radiation resistance from the primary source to secondary sources, and $W_{0}$ is the sound radiation power of the primary source only. The elements of the matrixes in Eq. (4) are $A_{i j}=0.5 Z_{0} \operatorname{sinc}\left(k d_{\mathrm{ss}, i j}\right), b_{i}=0.5 Z_{0} Q_{\mathrm{p}} \operatorname{sinc}\left(k d_{\mathrm{ps}}\right)$, and $W_{0}=0.5 Z_{0} Q_{\mathrm{p}}{ }^{2}$, where $Z_{0}=\rho_{0} \omega^{2} / 4 \pi c_{0}$ is the selfradiation resistance of a monopole in free space, $d_{\mathrm{ss}, i j}$ is the distance between the $i$ th and $j$ th secondary sources, and $\operatorname{sinc}(x)=\sin (x) / x$.

If the total sound radiation power is the cost function, the optimal strength vector of secondary sources can be obtained from Eq. (4) as 


$$
\mathbf{Q}_{\mathrm{s}}^{\mathrm{w}}=-\mathbf{A}^{-1} \mathbf{b} .
$$

Because of even distribution of the secondary sources, the optimal strengths of all secondary sources are the same. So, the optimal strength of the $i$ th secondary source can be presented as

$$
Q_{\mathrm{s}, i}^{\mathrm{w}}=-Q_{\mathrm{p}} \frac{\operatorname{sinc}\left(k d_{\mathrm{ps}}\right)}{\sum_{j=1}^{M} \operatorname{sinc}\left(k d_{\mathrm{ss}, i j}\right)} .
$$

The noise reduction is defined as the difference between the sound power level without and with control

$$
N R=10 \log _{10} \frac{W_{0}}{W} .
$$

For minimizing the sum of squared sound pressure and total sound power, the noise reduction can be obtained, respectively, as

$$
\begin{aligned}
N R_{\mathrm{p}}= & -10 \log _{10}\left\{1+M \sum_{j=1}^{M} \operatorname{sinc}\left(k d_{\mathrm{ss}, i j}\right)\right. \\
& \times\left|\frac{e^{-\mathrm{j} k d_{\mathrm{pe}}}}{d_{\mathrm{pe}}} \sum_{j=1}^{M} \frac{\operatorname{sinc}\left(k d_{\mathrm{se}, j i}\right)}{d_{\mathrm{se}, j i}}\right|^{2} \\
& \left.-2 M \operatorname{sinc}\left(k d_{\mathrm{ps}}\right) \operatorname{Re}\left[\frac{e^{-\mathrm{j} k d_{\mathrm{pe}}}}{d_{\mathrm{pe}}} \sum_{j=1}^{M} \frac{\operatorname{sinc}\left(k d_{\mathrm{se}, j i}\right)}{d_{\mathrm{se}, j i}}\right]\right\}
\end{aligned}
$$

and

$$
N R_{\mathrm{w}}=-10 \log _{10}\left[1-M \frac{\operatorname{sinc}^{2}\left(k d_{\mathrm{ps}}\right)}{\sum_{j=1}^{M} \operatorname{sinc}\left(k d_{\mathrm{ss}, i j}\right)}\right],
$$

where $\operatorname{Re}[]$ indicates the real part of [].

The noise reduction $N R_{\mathrm{w}}$ is the maximum of the achievable noise reduction because the secondary source strength $Q_{\mathrm{s}, i}^{\mathrm{w}}$ is obtained by minimizing the sound power. If locations of the error microphones can be chosen carefully to make the secondary source strength $Q_{\mathrm{s}, i}^{\mathrm{p}}$ equal to $Q_{\mathrm{s}, i}^{\mathrm{w}}$, the noise reduction $N R_{\mathrm{p}}$ by minimizing the sum of squared sound pressure will approach $N R_{\mathrm{w}}$.

At sufficiently low frequencies, $Q_{\mathrm{s}, i}^{\mathrm{p}}$ and $Q_{\mathrm{s}, i}^{\mathrm{w}}$ in Eqs. (3) and (6) can be simplified to

$$
Q_{\mathrm{s}, i}^{\mathrm{p}}=-\frac{1}{d_{\mathrm{pe}}}\left(\sum_{j=1}^{M} \frac{1}{d_{\mathrm{se}, j i}}\right)^{-1} Q_{\mathrm{p}}
$$

and

$$
Q_{\mathrm{s}, i}^{\mathrm{w}}=-\frac{1}{M} Q_{\mathrm{p}}
$$

Equalizing Eq. (9a) to Eq. (9b), the optimal distance between the primary source and error microphones is

$$
d_{\mathrm{pe}}^{\mathrm{w}}=M\left[\sum_{j=1}^{M} \frac{1}{d_{\mathrm{se}, j i}}\right]^{-1} .
$$

For a compact system where the radius of the error microphone surface $d_{\mathrm{pe}}$ is smaller than the radius of the secondary source surface $d_{\mathrm{ps}}$, Eq. (10) turns to $d_{\mathrm{pe}}^{\mathrm{w}}=d_{\mathrm{se}, 11}$ $=d_{\mathrm{ps}}-d_{\mathrm{pe}}^{\mathrm{w}}$, and it can be obtained that $d_{\mathrm{pe}}^{\mathrm{w}}=0.5 d_{\mathrm{ps}}$ when $M=1$, i.e., the best location for the error microphone is at the middle point between the primary source and the secondary source.

For other $M$ larger than 1, because it is hard to obtain an exact analytical solution for the optimal distance of error microphones, its range is estimated by applying the following inequality in Eq. (10):

$$
d_{\mathrm{se}, j i}>d_{\mathrm{ps}}-d_{\mathrm{pe}}^{\mathrm{w}} \quad(i \neq j) .
$$

So it has

$$
d_{\mathrm{pe}}^{\mathrm{w}}>M\left[\sum_{j=1}^{M} \frac{1}{d_{\mathrm{ps}}-d_{\mathrm{pe}}^{\mathrm{w}}}\right]^{-1}=d_{\mathrm{ps}}-d_{\mathrm{pe}}^{\mathrm{w}}
$$

and

$$
d_{\mathrm{pe}}^{\mathrm{w}}>0.5 d_{\mathrm{ps}} .
$$

This indicates that the optimal locations of the error microphones between the primary source and the secondary sources are closer to the secondary sources for multi-channel systems.

If the secondary source strength $Q_{\mathrm{s}, i}^{\mathrm{p}}$ cannot be adjusted to approach $Q_{\mathrm{s}, i}^{\mathrm{w}}$ by optimizing the error microphone locations in the sound pressure control or the primary source is not a monopole, the optimal error microphone locations cannot be directly formulated; however, it can be obtained with simulations as shown in Sec. III.

The main discovery of this research is that the optimal locations of error microphones are found to be located between the primary source and secondary sources for multichannel active radiation control systems and these optimal locations, which are independent of frequency when the frequency is sufficiently low, move toward the secondary sources when the number of secondary sources increases.

\section{SIMULATIONS AND DISCUSSIONS}

The secondary sources and error microphones are at the vertexes of regular polyhedrons on two separate sphere surfaces. ${ }^{20}$ Figure 2 shows eight typical configurations considered in this paper for the secondary source distribution, where the red circle indicates a primary source, and the black stars stand for secondary sources. Each error microphone (not shown in the figure) is placed on the line connecting the primary source and one of the secondary sources.

Monopole, dipole, and combined sources are considered as the primary source in the simulations. The monopole primary source is a point source located at the coordinate origin 


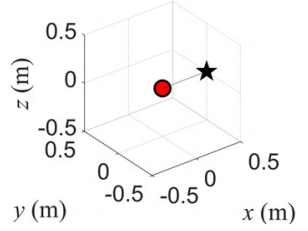

(a)

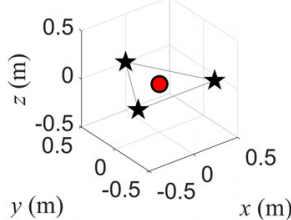

(c)

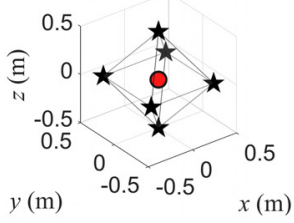

(e)

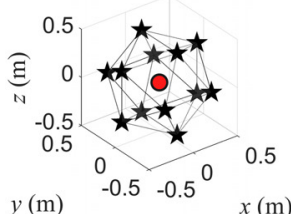

(g)

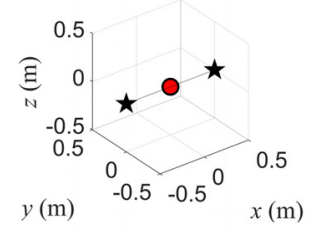

(b)

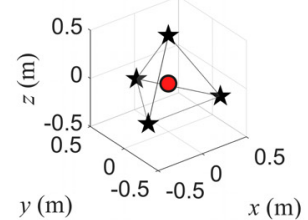

(d)

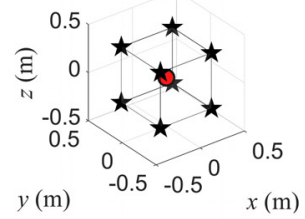

(f)

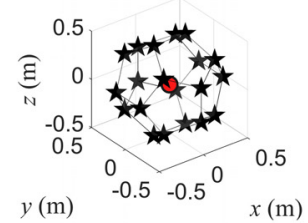

(h)
FIG. 2. (Color online) Typical distributions of secondary sources, the red circle indicates the primary source, the black stars stand for secondary sources, (a)-(h) are the systems with 1, 2, 3, 4, 6, 8, 12, and 20 secondary sources, respectively.

as shown in Fig. 1 with a strength of $1.0 \times 10^{-3} \mathrm{~m} / \mathrm{s}^{3}$. The dipole primary source consists of two point monopoles at $( \pm 0.005,0,0) \mathrm{m}$ with strengths of $-1.0 \times 10^{-3} \mathrm{~m} / \mathrm{s}^{3}$ and $1.0 \times 10^{-3} \mathrm{~m} / \mathrm{s}^{3}$, respectively. The combined primary source consists of three point monopoles at $(-0.005,0,0) \mathrm{m},(0,0$, $0) \mathrm{m}$, and $(0.005,0,0) \mathrm{m}$ with strengths of $-1.0 \times 10^{-3}$, $1.0 \times 10^{-3}$, and $1.0 \times 10^{-3} \mathrm{~m} / \mathrm{s}^{3}$, respectively. The secondary sources are placed at the sphere surface centered at $(0,0$, $0) \mathrm{m}$ with a radius of $0.5 \mathrm{~m}\left(d_{\mathrm{ps}}=0.5 \mathrm{~m}\right)$.

Figure 3 compares the noise reduction for the monopole primary source by minimizing the sound power and the sum of squared sound pressure at error microphones, which are optimally placed on the lines determined by the primary and secondary sources. The noise reduction by minimizing the sum of squared sound pressure, $N R_{p}$, and the noise reduction by minimizing the sound power, $N R_{\mathrm{w}}$, are almost the same when one secondary source is used. However, $N R_{\mathrm{p}}$ is smaller than $N R_{\mathrm{w}}$ at low frequencies when more secondary sources are employed because the noise reduction is sufficiently large and a small difference between the secondary source strength $Q_{\mathrm{s}, i}^{\mathrm{p}}$ and $Q_{\mathrm{s}, i}^{\mathrm{w}}$ will result in a large deviation of noise reduction. It is also found that $N R_{\mathrm{p}}$ and $N R_{\mathrm{w}}$ are more consistent at high frequencies and more different at low

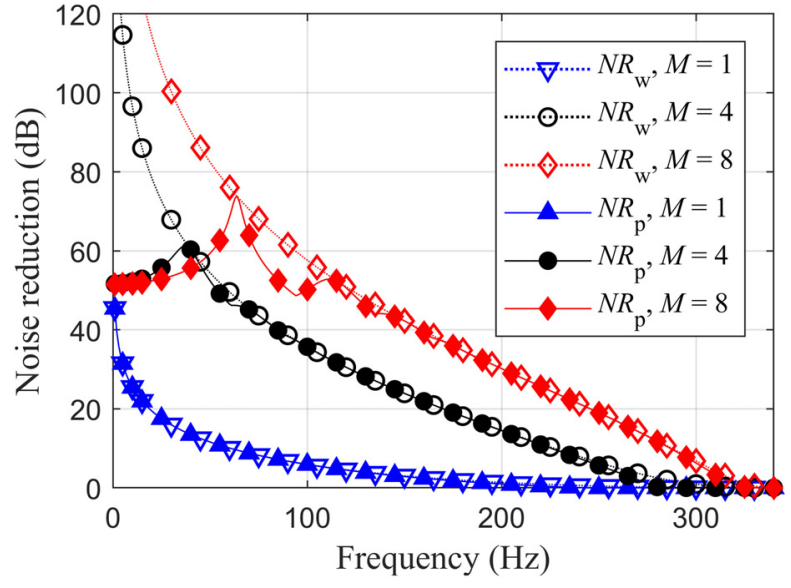

FIG. 3. (Color online) Noise reduction for the monopole primary source obtained from minimizing the sound power and from minimizing the sum of squared sound pressure at optimally placed error microphones.

frequencies when the number of secondary sources increases, and the reason is that $N R_{\mathrm{w}}$ increases faster with the secondary source number in low frequency range.

The noise reduction as a function of the distance between the primary source and error microphones is shown in Fig. 4 for different secondary source number $M$ by minimizing the sum of squared sound pressure at error microphones. For the monopole primary source at 63 and $125 \mathrm{~Hz}$, the noise reduction increases first and then decreases with the distance $d_{\mathrm{pe}}$ for the error microphones located inside the secondary source surface $\left(d_{\mathrm{pe}}<d_{\mathrm{ps}}\right)$. For the error microphones located outside the secondary source surface $\left(d_{\mathrm{pe}}\right.$ $\left.>d_{\mathrm{ps}}\right)$, the noise reduction increases gradually and converges to a constant value. Figures 4(c) and 4(d) illustrate similar results for the dipole and combined primary sources at $63 \mathrm{~Hz}$. The noise reduction has a largest extreme point when the error microphones are placed at the optimal locations between the primary source and secondary sources. For example, the optimal distance between the primary source and error microphones is $0.39 \mathrm{~m}$ when $M$ is 8 for the dipole primary source at $63 \mathrm{~Hz}$.

It can also be observed in Fig. 4 that the optimal locations of error microphones move toward secondary sources when the number of secondary sources increases. Figure 5 shows the optimal distance with the number of secondary sources at $63 \mathrm{~Hz}$, where the optimal distance is obtained by using both Eq. (10) and the simulations for the monopole primary source. Although the optimal distances are different for different primary sources when the secondary sources are not many, but they approach the same value when the number of secondary sources is greater than 5 . The optimal locations of error microphones are independent of the radiation pattern of the primary sources for multi-channel systems with sufficient secondary sources. In the figure, the optimal distance in simulations is larger than $0.25 \mathrm{~m}$ and converges to approximately $0.45 \mathrm{~m}$ for sufficient secondary sources. The optimal locations of error microphones are closer to the secondary sources than to the primary source as predicted in Sec. II.

The optimal distance as a function of frequency is shown in Fig. 6 for the monopole, dipole, and combined 


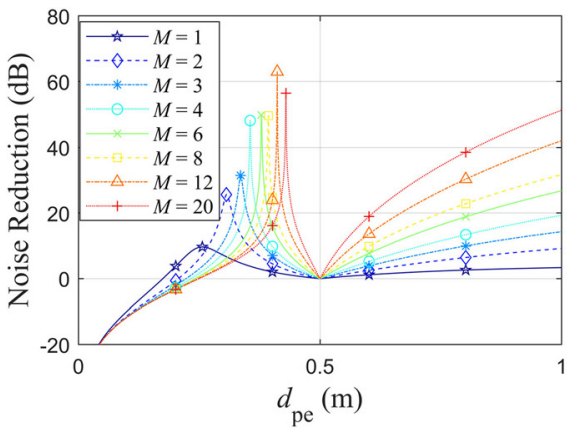

(a)

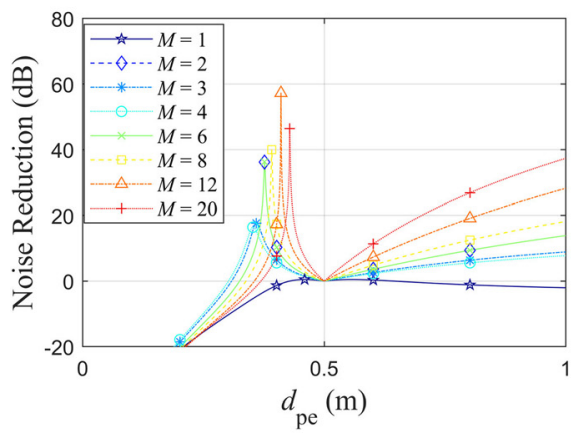

(c)

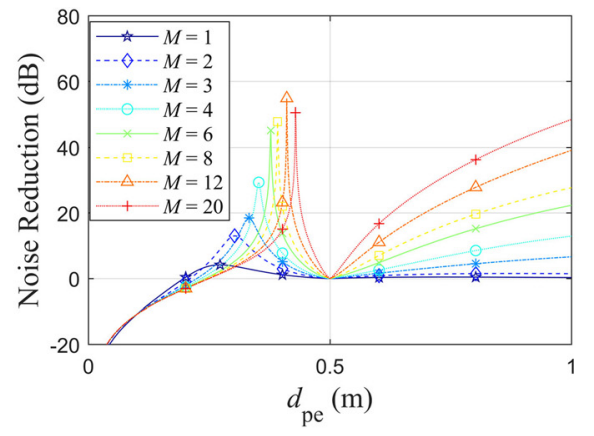

(b)

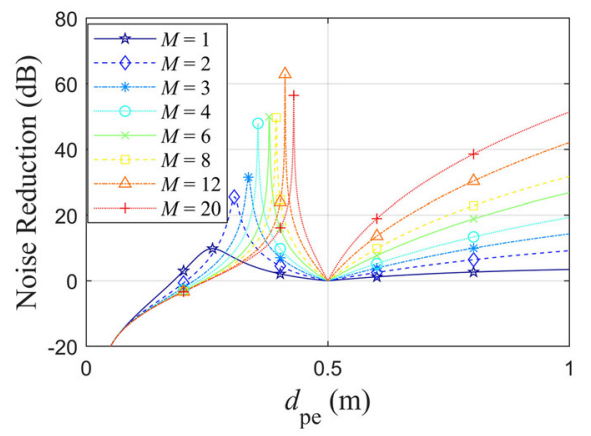

(d)
FIG. 4. (Color online) Noise reduction as a function of the distance between the primary source and error microphones for different secondary source number $M$ by minimizing the sum of squared sound pressure (a) monopole primary source at $63 \mathrm{~Hz}$, (b) monopole primary source at $125 \mathrm{~Hz}$, (c) dipole primary source at $63 \mathrm{~Hz}$, (d) combined primary source at $63 \mathrm{~Hz}$. primary sources with different number of secondary sources. The optimal distance increases with the number of secondary sources and varies little for different types of primary sources in low frequency as shown in Fig. 5. The optimal distance remains nearly constant at low frequency when the number of secondary sources is sufficiently large. For example, the variation ratio of the averaged optimal distance below $250 \mathrm{~Hz}$ for three different primary sources is within $2 \%$ when eight secondary sources are used.

In addition to the compact primary sources considered above, large extended primary sources are also numerically investigated. The interval between adjacent elements in dipole and combined primary sources increases to $0.2 d_{\mathrm{ps}}, 0.4 d_{\mathrm{ps}}$, and $0.6 d_{\mathrm{ps}}(0.1 \mathrm{~m}, 0.2 \mathrm{~m}$, and $0.3 \mathrm{~m})$, and a compound primary source consisting of eight monopoles on the vertexes of a cube

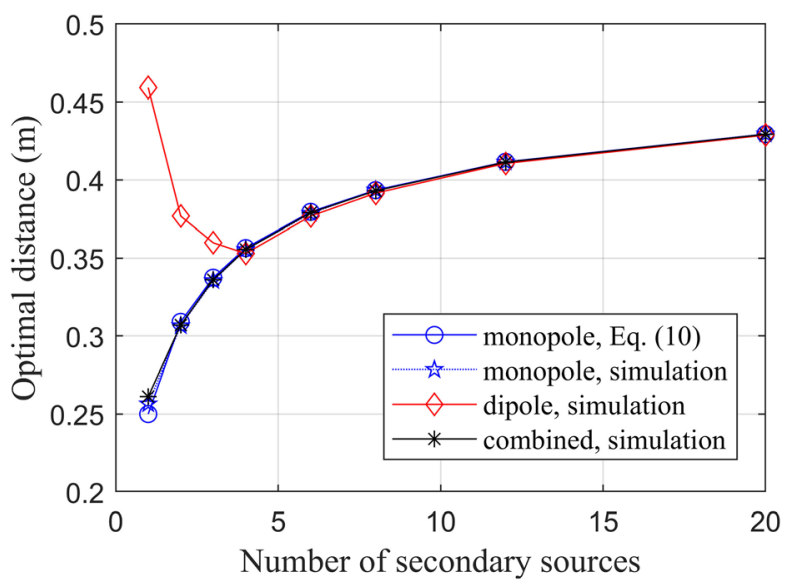

FIG. 5. (Color online) Optimal location of the error microphones with different numbers of secondary sources by minimizing the sum of squared sound pressure at $63 \mathrm{~Hz}$. centered at the coordinate origin is also employed. The strength of each monopole in the compound source is randomly chosen from $\left(0,1.0 \times 10^{-3}\right) \mathrm{m} / \mathrm{s}^{3}$. Although the radiation directivity of these primary sources becomes quite complicated, the simulation results show similar trends of the optimal distance of error microphones. As shown in Fig. 7, the optimal error microphone distance is around $0.38 \mathrm{~m}$ at $250 \mathrm{~Hz}$ when eight secondary sources are used to reduce the radiation from the extended dipole primary source with an element interval of $0.1 \mathrm{~m}$.

For all these multi-channel active radiation control systems, there exist optimal locations for the error microphones between the primary source and secondary sources, which remain unchanged at low frequencies despite the radiation pattern and geometry size of the primary source, and move

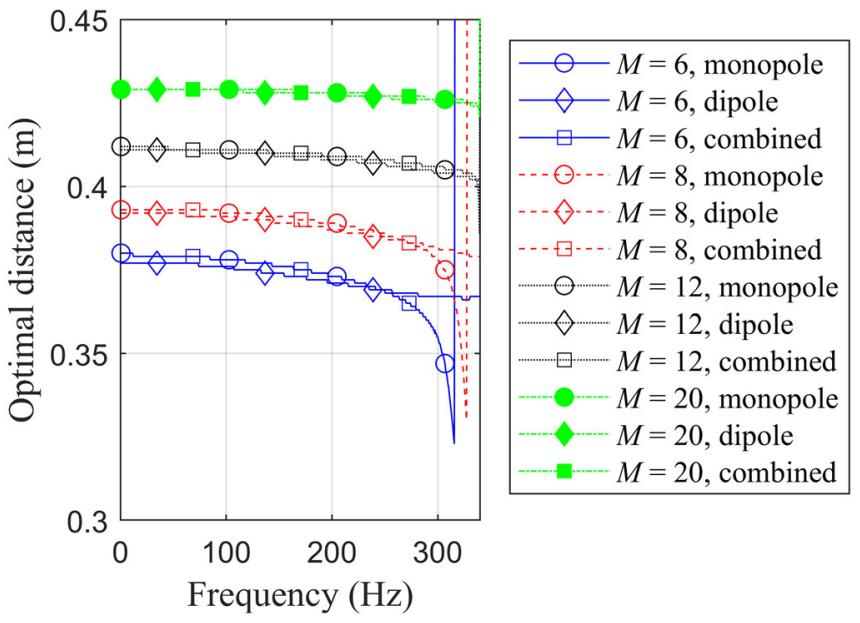

FIG. 6. (Color online) Optimal distance of the error microphones at different frequencies by minimizing the sum of squared sound pressure. 


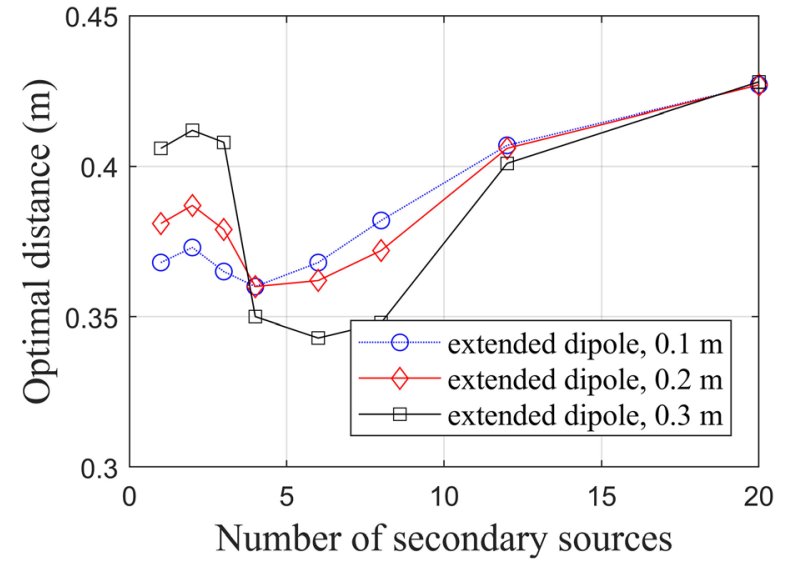

(a)

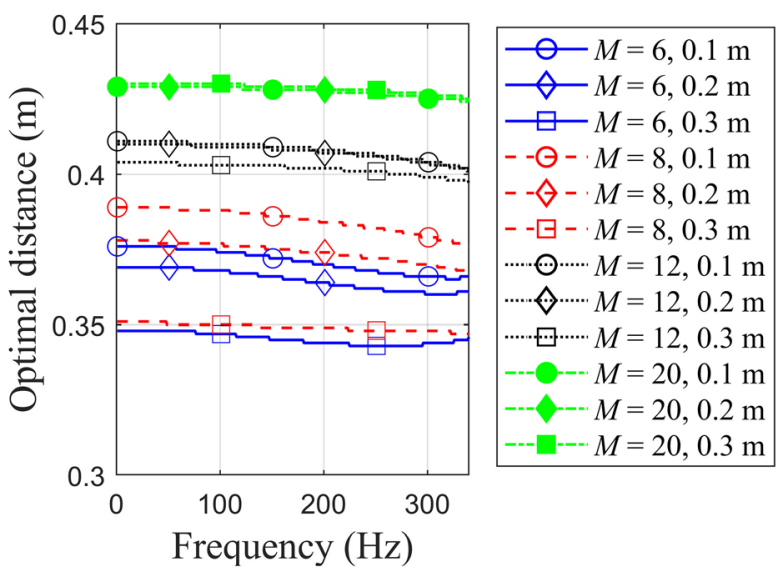

(b)

FIG. 7. (Color online) Optimal distance of the error microphones for the extended dipole sources with different element intervals (a) at $250 \mathrm{~Hz}$, (b) at different frequencies.

toward the secondary sources when the secondary source number increases. To explore the mechanism for this, the extended dipole primary source centered at the coordinate origin with an element interval of $0.1 \mathrm{~m}$ is taken as an example. In the system, eight secondary sources are arranged with $d_{\mathrm{ps}}=0.5 \mathrm{~m}$ according to Fig. 2(f) and the frequency of interest is $250 \mathrm{~Hz}$.

Figure 8 shows the sound pressure level (SPL) without and with control on the plane $y=x$, when the total sound power is used as the cost function. The projection of the primary source is marked as solid circles and 4 of 8 secondary sources on the plane $y=x$ are denoted as solid squares. The secondary sources are monopole point sources, so they radiate both outward and inward on their sphere surface. Therefore, some regions with very low sound pressure are generated between the primary source and secondary sources when the sound pressure level outside the secondary source surface is reduced together with the total sound radiation power.

The regions with low residual sound pressure inside the secondary source sphere depend on the arrangement of the secondary sources as shown in Fig. 8(b). When the lines connecting the primary and the secondary sources cross these regions, the locations with the lowest sound pressure

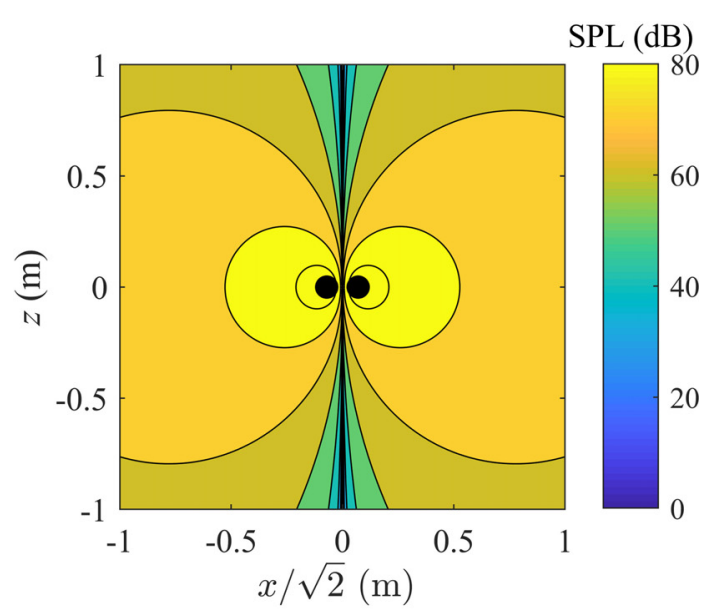

(a)

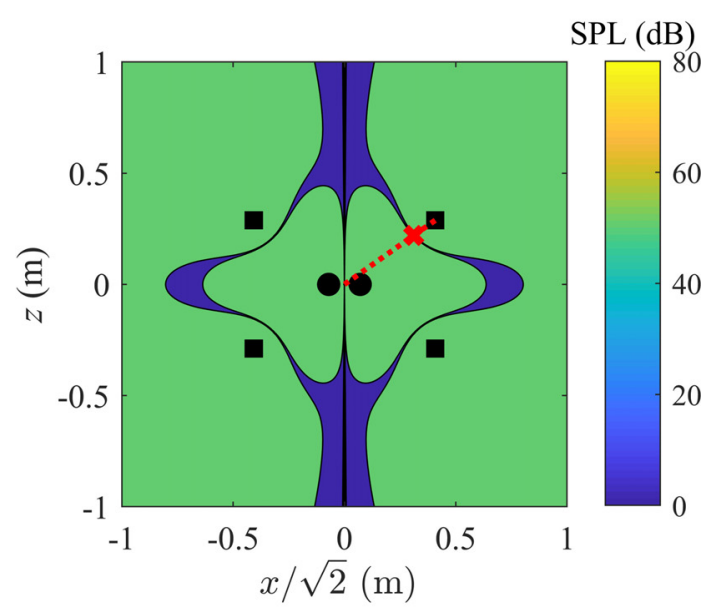

(b)

FIG. 8. (Color online) Sound pressure distribution on the plane $y=x$ for the extended dipole source with eight secondary sources at $250 \mathrm{~Hz}$ (a) without control, (b) with sound power control (the solid circles stand for the projection of primary source and the solid squares are secondary sources).

level are the optimal locations of the error microphones in the minimization of the sum of squared sound pressure. The distance between such a location marked as " $x$ " and the origin in Fig. 8(b) is $0.38 \mathrm{~m}$, which is exactly the optimal distance in the sound pressure minimization as shown in Fig. 7.

Figure 9(a) shows the averaged secondary source strength $(1 / M) \Sigma_{i=1}^{M}\left|Q_{\mathrm{s}, i}^{\mathrm{w}}\right|$ as a function of the secondary source number, where the averaged secondary source strength decreases with the secondary source number when more than four secondary sources are employed. The regions with low residual sound pressure in sound power minimization move toward the secondary sources and the optimal distance of error microphones for minimizing the sum of squared sound pressure increases accordingly. For example, the averaged secondary source strength decreases from $1.45 \times 10^{-4} \mathrm{~m} / \mathrm{s}^{3}$ to $0.51 \times 10^{-5} \mathrm{~m} / \mathrm{s}^{3}$ in Fig. 9(a) and the optimal distance of error microphones increases from 0.38 to $0.43 \mathrm{~m}$, respectively, when the secondary source number increases from 8 to 20 . 


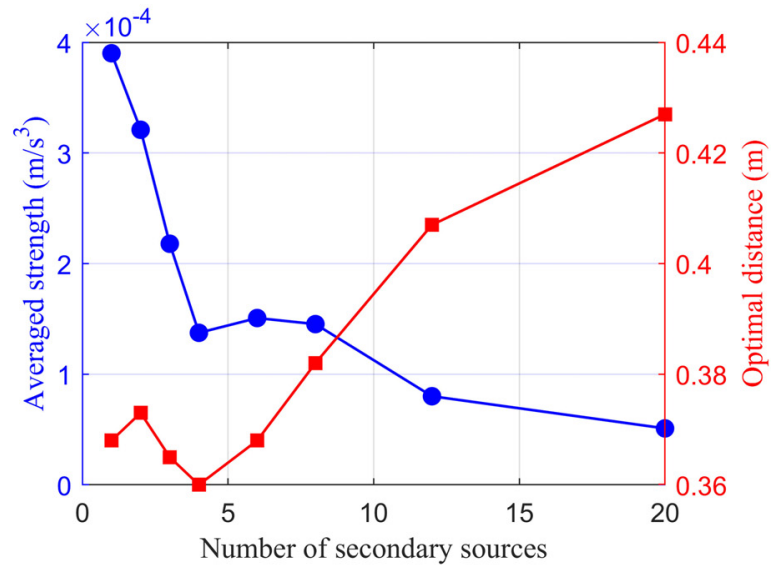

(a)

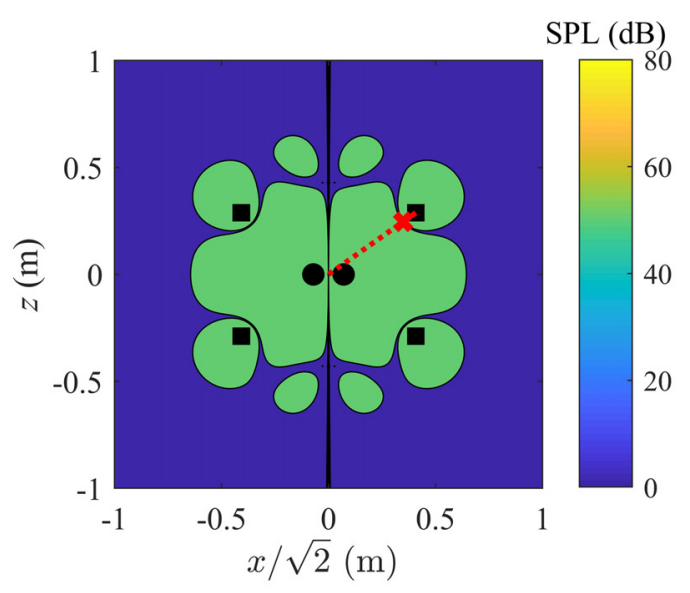

(b)

FIG. 9. (Color online) (a) Averaged secondary source strength in sound power minimization and the optimal distance of error microphones in sound pressure minimization as a function of secondary source number and (b) the sound pressure distribution on the plane $y=x$ with 20 secondary sources, for the extended dipole primary source at $250 \mathrm{~Hz}$ (the solid circles stand for the projection of primary source and the solid squares are secondary sources).

\section{EXPERIMENTS}

Experiments were conducted in the anechoic chamber of Nanjing University. Figure 10 shows the experimental setup, where the primary source is hanged by a vertical steel holder, the secondary loudspeakers are placed $0.5 \mathrm{~m}$ away from the primary source, and ten observation microphones were placed on a frame with a radius of $1.5 \mathrm{~m}$ to measure the radiated sound power according to ISO $3744 .{ }^{21}$ The B\&K $3560 \mathrm{D}$ analyzer was used to drive the primary source and to provide the reference signal for the Tiger ANC WIFI-M controller embedded with the waveform synthesis algorithm. ${ }^{22}$

The primary source and secondary sources are custommade close-box loudspeakers with the diameter of the loudspeakers being $2.54 \mathrm{~cm}$. Two loudspeakers were placed $20 \mathrm{~cm}$ away to constitute the dipole primary source, and three loudspeakers were placed in a line with an interval of $10 \mathrm{~cm}$ to constitute the combined primary source. Consider the radiation efficiency of the loudspeakers, the test frequency was chosen as $250 \mathrm{~Hz}$. The measured radiation

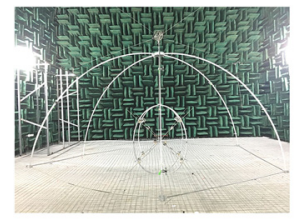

(a)

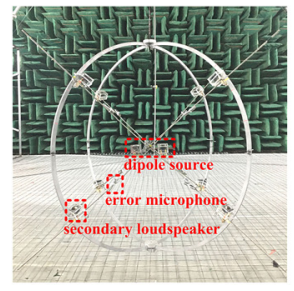

(c)

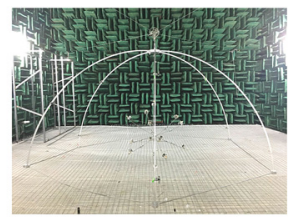

(b)

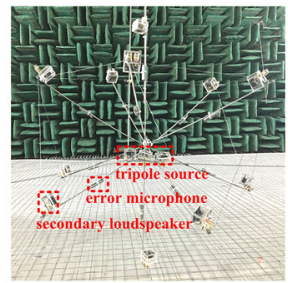

(d)
FIG. 10. (Color online) Experimental setup: (a) an overview of the 8channel experiments, (b) an overview of the 12-channel experiments, (c) the 8-channel system for the dipole primary source, (d) the 12-channel system for the combined primary source.

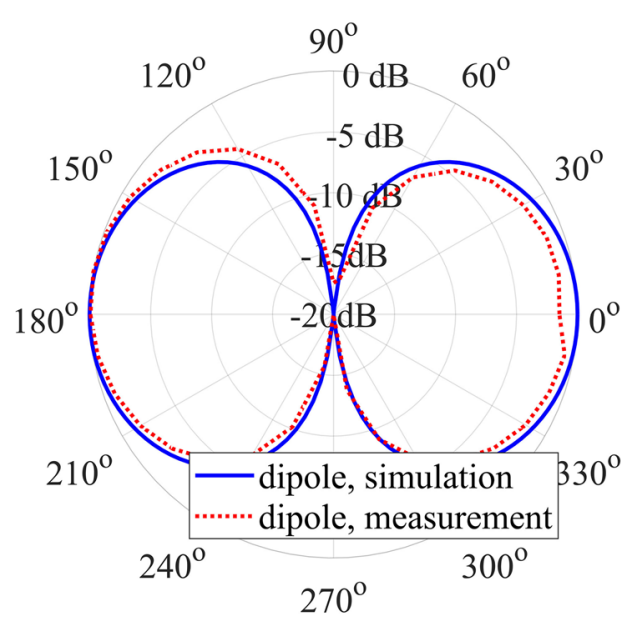

(a)

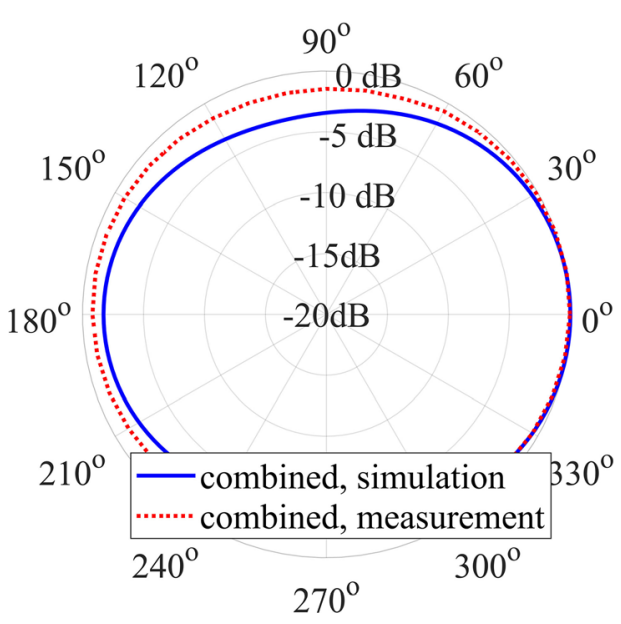

(b)

FIG. 11. (Color online) Directivity of the primary source at $1 \mathrm{~m}$ away: (a) dipole primary source, (b) combined primary source. 
directivity of the primary sources agrees well with the simulation results as shown in Fig. 11, where the maximal SPL deviation is about $2 \mathrm{~dB}$.

Both 8-channel and 12-channel systems were employed in the experiments. The secondary loudspeakers were arranged according to Figs. 2(f) and 2(g) and the experiment setup is shown in Figs. 10(c) and 10(d). The error microphones were on the lines connecting the primary source and their corresponding secondary loudspeakers, and the distance between each error microphone and the center of the primary source was chosen from 0.25 to $0.75 \mathrm{~m}$ with an interval of $0.05 \mathrm{~m}$.

Figure 12 compares the measured sound power level with and without control, where the maximal difference between the simulation results and measured results is within $5 \mathrm{~dB}$. In Fig. 12(a), the maximum of the measured noise reduction of the 8channel systems occurs at $d_{\mathrm{pe}}=0.35 \mathrm{~m}$ as calculated for both the dipole and combined primary sources, while in Fig. 12(b), the maximum of the measured noise reduction of the 12channel systems occurs at $d_{\mathrm{pe}}=0.35 \mathrm{~m}$ which is close to the simulation result of $0.375 \mathrm{~m}$. This difference between the simulation and experiment results is caused by the inaccurate

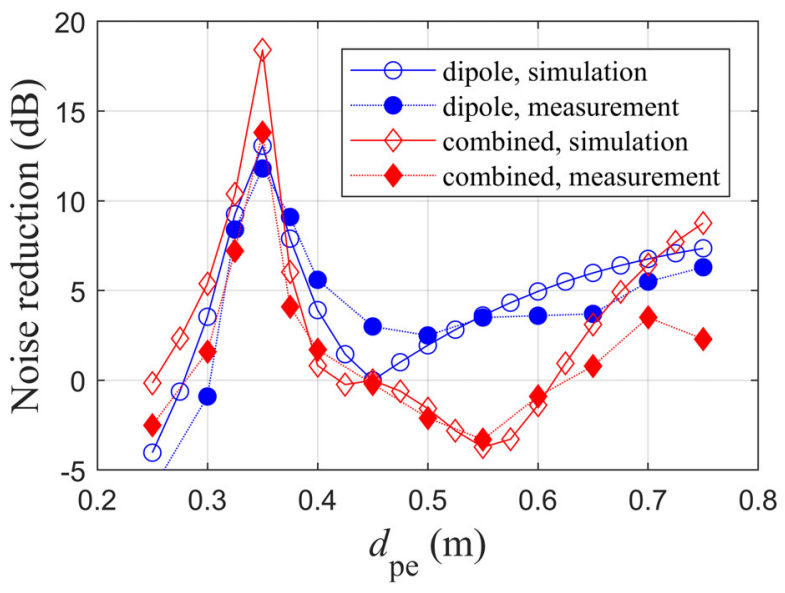

(a)

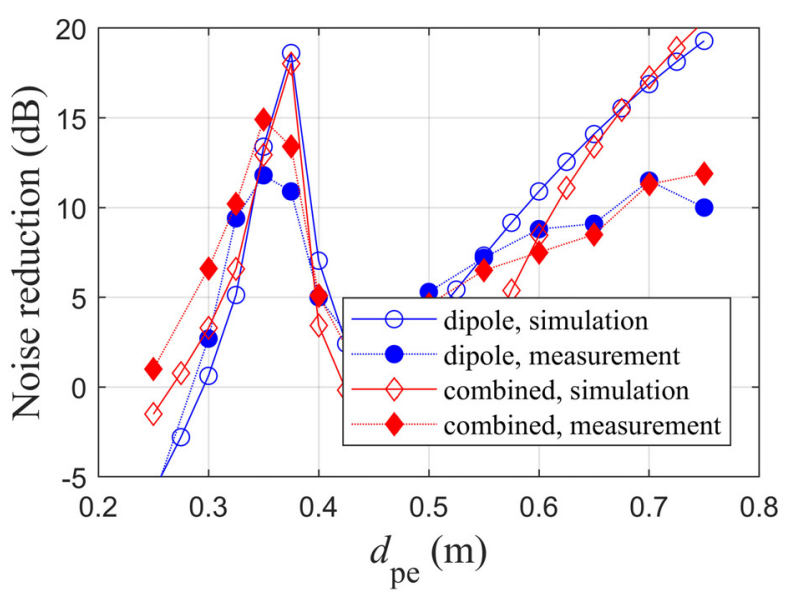

(b)

FIG. 12. (Color online) Noise reduction of the dipole and combined primary sources with different error microphone placements: (a) 8-channel systems, (b)12-channel systems. distance between the error microphone and the primary sources in the experiments. The experimental results with the 8channel and 12-channel active radiation control systems for the dipole and combined primary sources validate the discoveries from the simulations.

\section{CONCLUSIONS}

For a multi-channel active radiation control system which has secondary sources distributed uniformly on a sphere surface surrounding the primary source, the optimal locations of error sensors for minimizing the sum of squared sound pressure is found to be between the primary source and the secondary sources. Both simulation and experiment results show that the optimal locations of error microphones are independent of the type of primary source when the number of secondary sources are many, which remain unchanged in low frequency range and move toward the secondary sources when the secondary source number increases. Therefore, for active radiation control applications in low frequency range, a compact multi-channel system can be developed by locating the error microphones between the primary source and the secondary sources. Future work will investigate the behavior of the optimal locations with the existence of reflecting surfaces.

\section{ACKNOWLEDGMENTS}

This research was supported by the National Science Foundation of China (Grant Nos. 11874218 and 11874219) and under the High-tech Ship Research Projects funding scheme of the Ministry of Industry and Information Technology of China. The authors would also like to thank Mr. Jiaxin Zhong for his help in preparing Fig. 1.

${ }^{1} \mathrm{~N}$. Hesselmann, "Investigation of noise reduction on a $100 \mathrm{kVA}$ transformer tank by means of active methods," Appl. Acoust. 11(1), 27-34 (1978).

${ }^{2}$ K. L. Gee and S. D. Sommerfeldt, "Application of theoretical modeling to multichannel active control of cooling fan noise," J. Acoust. Soc. Am. 115, 228-236 (2004).

${ }^{3}$ B. B. Monson, S. D. Sommerfeldt, and K. L. Gee, "Improving compactness for active noise control of a smallaxial cooling fan," Noise Control Eng. J. 55, 397-407 (2007).

${ }^{4} \mathrm{Q}$. Guo, J. Tao, G. Jin, and X. Qiu, "Physical system optimization in global active radiation control with space constraint," in Proceedings of the 46th Inter-Noise, Hong Kong (2017).

${ }^{5}$ C. H. Hansen, S. Snyder, X. Qiu, L. Brooks, and D. Moreau, Active Control of Noise and Vibration, 2nd ed. (CRC Press, Boca Raton, 2012).

${ }^{6} \mathrm{~B}$. T. Wang and C. R. Fuller, "Near-field pressure, intensity, and wavenumber distributions for active structural acoustic control of plate radiation: Theoretical analysis," J. Acoust. Soc. Am. 92, 1489-1498 (1992).

${ }^{7}$ X. Qiu, C. H. Hansen, and X. Li, "A comparison of near-field acoustic error sensing strategies for the active control of harmonic free field sound radiation," J. Sound Vib. 215, 81-103 (1998).

${ }^{8}$ A. Berry, X. Qiu, and C. H. Hansen, "Near-field sensing strategies for the active control of the sound radiated from a plate," J. Acoust. Soc. Am. 106, 3394-3406 (1999).

${ }^{9}$ X. Li, X. Qiu, C. H. Hansen, and Y. Ai, "Active control of sound radiation from a small transformer using near-field sensing," Int. J. Acoust. Vib. 7(2), 85-92 (2002).

${ }^{10} \mathrm{X}$. Li, X. Qiu, and C. H. Hansen, "Active control of sound radiation by structures using near-field sensing strategies," Int. J. Acoust. Vib. 7(2), 93-99 (2002). 
${ }^{11}$ S. Wang, H. Sun, J. Pan, and X. Qiu, "Near-field error sensing for active directivity control of radiated sound," J. Acoust. Soc. Am. 144, 598-604 (2018).

${ }^{12}$ T. Hayashi, A. Enamito, and S. Suzuki, "Active acoustic power control of a single primary and two secondary loudspeakers by the acoustic nodal point method," J. Acoust. Soc. Jpn. 16(4), 213-221 (1995).

${ }^{13}$ B. M. Shafer, K. L. Gee, and S. D. Sommerfeldt, "Verification of a nearfield error sensor placement method in active control of compact noise sources," J. Acoust. Soc. Am. 127, EL66-EL72 (2010).

${ }^{14}$ T. Martin and A. Roure, "Active noise control of acoustic sources using spherical harmonics expansion and a genetic algorithm simulation and experiment," J. Sound Vib. 212(3), 511-523 (1998).

${ }^{15}$ J. Garcia-Bonito, S. J. Elliott, and C. C. Boucher, "Generation of zones of quiet using a virtual microphone arrangement," J. Acoust. Soc. Am. 101(6), 3498-3516 (1997).

${ }^{16}$ A. P. Berkhoff, "Control strategies for active noise barriers using nearfield error sensing," J. Acoust. Soc. Am. 118(3), 1469-1479 (2005).
${ }^{17}$ C. D. Peterson, A. C. Zander, B. S. Cazzolato, and C. H. Hansen, “A moving zone of quiet for narrowband noise in a one-dimensional duct using virtual sensing," J. Acoust. Soc. Am. 121(3), 1459-1470 (2007)

${ }^{18}$ C. R. Fuller, C. Papenfuss, and T. D. Saux, "Active-passive control of portable generator set radiated noise," in Proceedings of Acoustics 2012, Fremantle, Australia (2012).

${ }^{19}$ M. Krzysztof and M. Pawelczyk, "Virtual microphone control for a lightweight active noise-reducing casing," in Proceedings of ICSV23, Athens, Greece (2016)

${ }^{20} \mathrm{P}$. Nelson and S. Elliott, Active Control of Sound (Academic Press, New York, 1992).

${ }^{21}$ ISO 3744: 1994, “Acoustics-Determination of sound power levels of noise sources using sound pressure-Engineering method in an essentially free field over a reflecting plane" (International Organization for Standardization, Geneva, Switzerland, 1994).

${ }^{22} \mathrm{X}$. Qiu and C. Hansen, "An algorithm for active control of transformer noise with on-line cancellation path modelling based on the perturbation method," J. Sound Vib. 240(4), 647-665 (2001). 